\title{
MAXIMUM MODULUS ALGEBRAS AND ANALYTIC VARIETIES
}

\author{
DONNA KUMAGAI AND ZBIGNIEW SLODKOWSKI
}

(Communicated by Paul S. Muhly)

\begin{abstract}
Let $A$ be a maximum modulus algebra on $X$, and $V$ a maximal open subset of $X$ such that $V$ has the structure of one-dimensional variety on which functions from $A$ are analytic. Then, the restriction algebra $A_{X \backslash V}$ is again a maximum modulus algebra.
\end{abstract}

Let $X$ be a locally compact space, and $A$ a complex algebra of continuous functions on $X$ that contains constant functions and separates points of $X$. $A$ is called a maximum modulus algebra on $X$ if the following local maximum property holds: Given a compact subset $N$ of $X$, for each $x_{0} \in N$ and each $g \in A$,

$$
\left|g\left(x_{0}\right)\right| \leq \max _{\partial_{x} N}|g|
$$

The algebra of all analytic functions on a complex manifold is an example of a maximum modulus algebra. If we let $A$ be a uniform algebra defined on its maximal ideal space $M$ and $X=M \backslash S(A)$, where $S(A)$ is the Silov boundary of $M$, then $A$ restricted to $X$ is a maximum modulus algebra. Conditions for existence of analytic structures associated with maximum modulus algebras have been researched extensively. See [6] for some notable results.

In the present study we show that if $A$ is a maximum modulus algebra on $X$ then the existence of an open analytic variety $V$ in $X$ guarantees that the restriction algebra $A_{X \backslash V}$ on the complement of $V$ in $X$ is again a maximum modulus algebra. Our method is to apply several previous results by Z. Slodkowski in [4], where he develops a higher dimensional version of Oka's analytic multivalued functions. These results in [4] are combined with a now classic theorem on analytic structure by Aupetit and Wermer [1]. An interesting result that relates to ours is obtained by T. W. Gamelin [2] in the context of uniform algebras using Hayashi's pole function.

We begin with some preliminary expositions.

Definition. Let $X$ and $Y$ be topological spaces. A continuous map $G: X \rightarrow Y$ is said to be proper if $G^{-1}(K)$ is compact in $X$ for every compact $K \subset Y$.

Received by the editors October 27, 1989 and, in revised form, February 12, 1990.

1980 Mathematics Subject Classification (1985 Revision). Primary 46J10; Secondary 32E25. 
We make the following remarks on proper maps for later use.

Remark (i). Let $N$ be a compact subset of $X$ and $G: X \rightarrow Y$ be continuous. Then, the restriction of $G$ to $N$ is proper onto its range.

Remark (ii). Let $G: X \rightarrow Y$ be continuous and $N \subset X$ compact. Then, the restriction map $\left.G\right|_{N \backslash G^{-1}(G[\partial N])}$ is proper. In general, the restriction of a proper map to the full inverse image of a set is proper.

Proof of (ii). Put $U=N \backslash G^{-1}(G[\partial N])$. Then $G(U) \cap G(\partial N)=\varnothing$. Let $K \subset$ $G(U)$ be compact. To show that $G^{-1}(K)$ is compact it suffices to show that it is closed in $N$. So let $\left\{k_{\alpha}\right\}$ be a net in $G^{-1}(K)$ with $k_{\alpha} \rightarrow k \in N$. Since $G$ is continuous, $G\left(k_{\alpha}\right) \rightarrow G(k)$. Since $K$ is closed, $G(k) \in K$, i.e., $k \in G^{-1}(K)$.

Remark (iii). Let $X$ and $Y$ be regions in $\mathbf{C}^{n}$ and $\mathbf{C}^{k}$ respectively. Suppose that $G: X \rightarrow Y$ is holomorphic and proper. For each $w \in Y$, the fiber $G^{-1}(w)=\{\lambda \in X \mid G(\lambda)=w\}$ is finite.

Proof of (iii). If $w=\left(w_{1}, w_{2}, \ldots, w_{k}\right) \in Y$ and $G=\left(g_{1}, \ldots, g_{k}\right)$, then $G^{-1}(w)$ is the intersection of the zero sets of $g_{i}-w_{i}, 1 \leq i \leq k$, and is compact since $G$ is proper. By a well-known theorem, any compact subvariety of $\mathbf{C}^{n}$ is a finite set.

Remark (iv). A composition of proper maps is proper.

Remark (v). Let $G: X \rightarrow \mathbf{C}^{n}$ be proper onto its range. Then, $G(X)$ is locally closed; i.e., there exists an open subset $U$ of $\mathrm{C}^{n}$ with $G(X) \subseteq U$ such that $\overline{G(X)} \cap U=G(X)$.

Proof of (v). For each $y$ in $G(X)$ choose a neighborhood $T_{y}$ of $y$ such that $G^{-1}\left(\overline{T_{y}}\right)$ is compact. Let $U$ be a union of such neighborhoods $T_{y}$. We assert that $G$ is proper into $U$. Indeed, let $K \subset U$ be compact. It is covered by a finite set $\left\{T_{y_{1}}, \ldots, T_{y_{s}}\right\}$. Since $G^{-1}\left(\bar{T}_{y_{1}} \cup \cdots \cup \bar{T}_{y_{s}}\right)$ is compact and $G^{-1}(K)$ is closed, $G^{-1}(K)$ is compact. Next we show that $\overline{G(X)} \cap U=G(X)$. Given any $y \in \overline{G(X)} \cap U$, choose a neighborhood $T$ of $y$ in $U$ such that $\bar{T}$ and hence $G^{-1}(\bar{T})$ are compact. Then $G\left\{G^{-1}(\bar{T})\right\}=\bar{T} \cap G(X)$ is compact, and therefore closed. Since $y \in \overline{G(X)}$ and $T$ is a neighborhood of $y$, we have $y \in \overline{\bar{T} \cap G(X)}=\bar{T} \cap G(X) \subset G(X)$.

The first two lemmas are stated in a general setting of a locally compact system but they apply to our case as well. Recall that a pair $(X, A)$ is called a locally compact system in the sense of Rickart if $X$ is a locally compact space; $A$ a complex algebra of continuous functions on $X$ which contains constants and separates points of $X$; and the given topology on $X$ is the coarsest topology which makes functions in $A$ continuous.

Lemma 1. Let $(X, A)$ be a locally compact system and let $K$ be a compact subset of $X$. Given an open covering $\left\{U_{t}\right\}$ of $X$ and functions $g_{1}, \ldots, g_{r}$ 
from $A$, there exists an open subset $U$ of $X$ and functions $g_{1}, \ldots, g_{m}$ in $A$ such that

(i) $K \subset U$

(ii) $\left\{g_{1}, \ldots, g_{r}\right\} \subset\left\{g_{1}, \ldots, g_{m}\right\}$

(iii) The map $G=\left\{\left.g_{1}\right|_{U}, \ldots,\left.g_{m}\right|_{U}\right)$ is proper onto its range.

Furthermore, for every $y \in G(U)$ the fiber above $y, G^{-1}(y)$, is contained in some $U_{t}$.

Lemma 2. Let $L$ and $K$ be compact subsets of $\mathbf{R}^{n}$ with $L \subset K \subset \mathbf{R}^{n}$. Suppose $u: K \rightarrow[-\infty,+\infty)$ is upper semicontinuous and $\max u>\left.\max u\right|_{L}$. Then, there exists $x_{0} \in K \backslash L, \varepsilon>0$, and an affine form $l: R^{n} \rightarrow R$ such that

$$
\begin{gathered}
(u+l)\left(x_{0}\right)=0 \\
(u+l)(x) \leq-\varepsilon\left|x-x_{0}\right|^{2} \quad \text { for all } x \in K .
\end{gathered}
$$

See [5] for proofs of Lemmas 1 and 2.

Our main theorem is as follows:

Theorem 1. Let $A$ be a maximum modulus algebra on $X$. Let $V \subseteq X$ be a maximal open subset of $X$ such that $V$ has the structure of one dimensional variety on which all functions from $A$ are analytic. Denote $Y=X \backslash V$. Then the restriction algebra $A_{Y}$ is again a maximum modulus algebra.

For proof of Theorem 1 we need the following lemma.

Lemma 3. Let $\left(\mathbf{C}^{m}\right)^{*}, m>1$, be the set of complex linear functionals and let $M$ be a one-dimensional variety in $\mathbf{C}^{m}$. Let $S=\left\{f \in\left(\mathbf{C}^{m}\right)^{*}: f^{-1}(w) \cap M\right.$ is countable for all $w \in \mathbf{C}\}$. Then, $S$ is dense in $\left(\mathbf{C}^{m}\right)^{*}$. In fact, $S$ contains a dense $G_{\delta}$-set.

Proof of Lemma 3. Since $M$ is a one-dimensional variety, it is a union of at most countably many connected one-dimensional manifolds, $M_{1}, M_{2}, \ldots$, and of countable singular points. For each $M_{n}$, choose a pair of distinct points, $a_{n}, b_{n} \in M_{n}$. Let $S_{n}=\left\{f \in\left(\mathbf{C}^{m}\right)^{*}: f\left(a_{n}\right) \neq f\left(b_{n}\right)\right\}$. Observe that if $f \in$ $S_{n}$ then for each $w$ in the range of $f$, the set $f^{-1}(w) \cap M_{n}$ is countable. Otherwise it would be a subvariety of $M_{n}$ containing a neighborhood. Since $M_{n}$ is a connected manifold, $f^{-1}(w) \supset M_{n}$, which implies that $\left.f\right|_{M_{n}}=$ constant, contrary to the assumption that $f\left(a_{n}\right) \neq f\left(b_{n}\right)$. Clearly, $S_{n}$ is an open dense set in $\left(\mathbf{C}^{m}\right)^{*}$ and $S_{n} \subset S$ for each $n$.

Proof of Theorem 1. Suppose $A_{Y}$ does not have the local maximum property, i.e., there is a function $g_{0} \in A$ and a compact subset $N$ of $X$ such that

$$
\max _{x \in N \cap Y}\left|g_{0}(x)\right|=1>\max _{x \in Y \cap \partial N}\left|g_{0}(x)\right|,
$$

where $\partial N$ is the topological boundary of $N$ in $X$. Let $\lambda$ be a point in $N \cap Y$ on which $\left|g_{0}\right|$ attains its maximum. Multiplying by a suitable constant we 
may alter $g_{0}$ so that $g_{0}(\lambda)=\left|g_{0}(\lambda)\right|=1$. Denote $J=\left\{x \in N: g_{0}(x)=1\right\}$. Note that the map $x \rightarrow g_{0}(x): N \cap Y \backslash g_{0}^{-1} g_{0}(\partial N \cap Y) \rightarrow \mathrm{C}$ is proper onto its range. By Lemma 1 we can find functions $g_{1}, \ldots, g_{m}$ in $A$ such that $G=\left(g_{0}, g_{1}, \ldots, g_{m}\right)$ is a proper map of some neighborhood of $J$ in $X$ onto its range. Without loss of generality we can shrink $X$ and assume that $X$ is already such a neighborhood. In view of Remark ( $\mathrm{v}$ ) on proper maps made earlier we see that $G(X)$ is locally closed and so is $G(Y)$; i.e., there is an open set $U \supset G(X)$ such that $G(X)=U \cap \overline{G(X)}$ and $G(Y)=U \cap \overline{G(Y)}$. Denote by $\xi_{0}, \xi_{1}, \ldots, \xi_{m}$ the coordinate functionals on $\mathbf{C}^{m+1}$. Thus, if $z \in G(Y)$ then $\xi_{0}(z)=g_{0}(w)$ for some $w \in Y$. By Lemma 2 there exists $z_{0} \in G(J), \varepsilon>0$, and a $\mathbf{C}$-linear form $l: \mathbf{C}^{m+1} \rightarrow \mathbf{C}$ such that

$$
\begin{gathered}
\left(\operatorname{Re} \xi_{0}+\operatorname{Re} l\right)\left(z_{0}\right)=0 \\
\left(\operatorname{Re} \xi_{0}+\operatorname{Re} l\right)(z) \leq-\varepsilon\left|z-z_{0}\right|^{2} \text { for all } z \in G(Y) .
\end{gathered}
$$

It holds that

$$
\left(\operatorname{Re} \xi_{0}+\operatorname{Re} l\right)\left(z_{0}\right)>\left(\operatorname{Re} \xi_{0}+\operatorname{Re} l\right)(z) \quad z \in G(Y) \backslash z_{0} .
$$

Choose an open neighborhood $H$ of $z_{0}$ such that $\bar{H} \subseteq U$ and $\bar{H}$ is compact. Then, there exists $\varepsilon>0$ such that $\left(\operatorname{Re} \xi_{0}+\operatorname{Re} l\right)\left(z_{0}\right)>2 \varepsilon+\left(\operatorname{Re} \xi_{0}+\operatorname{Re} l\right)(z)$, $z \in \partial H \cap G(Y)$.

If we put $V^{*}=G(X) \backslash G(Y)$ then it is a one-dimensional variety, since it is the proper image of the one-dimensional variety $V \cap G^{-1}\left(V^{*}\right)$ under the map $\left.G\right|_{G^{-1}\left(V^{*}\right)}$. By Lemma 3, now we find a linear functional $f$ with the properties that (i) $f^{-1}(w) \cap V^{*}$ is at most countable for all $w \in \mathbf{C}$ and (ii) $\max _{z \in \bar{H} \cap G(Y)}\left|\left(\xi_{0}+l\right)(z)-f(z)\right|<\varepsilon$. Consequently, $\left.\max \operatorname{Re} f\right|_{H \cap G(Y)}>$ $\left.\max \operatorname{Re} f\right|_{\partial H \cap G(Y)}$. Choose a point $z^{*}$ in $H \cap G(Y)$ at which $\operatorname{Re} f$ attains its maximum. If we put $w^{*}=f\left(z^{*}\right)$ then $f^{-1}\left(w^{*}\right) \cap V^{*}$ is at most countable by the choice of $f$. Observe that $f$ restricted to the set $\bar{H} \cap G(Y)$ is proper onto its range and so is $f$ restricted to the set $\left\{z \in H \cap G(Y): \operatorname{Re} f(z)>\left.\max \operatorname{Re} f\right|_{\partial H}\right\}$ by Remark (ii) on proper maps. By this there is $\delta_{0}$ such that $f$ restricted to the intersection of $[G(Y) \cap \bar{H}]$ and $f^{-1}\left\{w:\left|w-f\left(z^{*}\right)\right|<\delta_{0}\right\}$ is a proper map. Consequently, $f^{-1}\left(w^{*}\right) \cap G(Y)$ is compact. Since $V^{*}=G(X) \backslash G(Y)$, we see that $f^{-1}\left(w^{*}\right) \cap G(X)$ is a disjoint union of a compact set $f^{-1}\left(w^{*}\right) \cap G(Y)$ and a countable set $f^{-1}\left(w^{*}\right) \cap V^{*}$. We shall construct a relatively compact neighborhood $B_{0}$ of $f^{-1}\left(w^{*}\right) \cap G(Y)$ in the hyperplane $f^{-1}\left(w^{*}\right)$ such that $B_{0} \subset U$ and $\partial B_{0}$ is disjoint from $f^{-1}\left(w^{*}\right) \cap G(X)$. For each $x \in f^{-1}\left(w^{*}\right) \cap G(Y)$ the set of distances $\left\{d_{n}(x)=\left|x-a_{n}\right|: a_{n} \in f^{-1}\left(w^{*}\right) \cap V^{*}\right\}$ is countable. Pick $\varepsilon(x)>0$ such that $\varepsilon(x) \neq d_{n}(x)$ for any $n$ and the ball of radius $\varepsilon(x)$ about $x$ is contained in $U$. Cover $f^{-1}\left(w^{*}\right) \cap G(Y)$ with a finite union of these balls and call it $B_{0}$. Clearly, $\partial B_{0}$ does not meet $f^{-1}\left(w^{*}\right) \cap G(Y)$ nor $f^{-1}\left(w^{*}\right) \cap V^{*}$, i.e., $\partial B_{0} \cap f^{-1}\left(w^{*}\right) \cap G(X)=\varnothing$. Let $e$ be a unit vector orthogonal to the hyperplane 
$f^{-1}\left(w^{*}\right)$. Since $G(X)$ is closed in $U$ and $\partial B_{0}$ is compact, there is $\delta<\delta_{0}$ such that $\bigcup_{|w|<\delta}\left(\partial B_{0}+w e\right)$ is disjoint from $G(X)$. Denote $B=\bigcup_{|w|<\delta}\left(B_{0}+w e\right)$. It is now clear that $f: B \cap G(X) \rightarrow C$ is proper onto its range. Therefore, by Remark (iii) about proper maps made earlier, for each $w$ in $\left\{\left|w-f\left(z^{*}\right)\right|<\delta\right.$, $\left.\operatorname{Re} w>\operatorname{Re} f\left(z^{*}\right)\right\}$, the set $f^{-1}(w) \cap B \cap G(X)$ is finite. Slodkowski showed in [4] that $\forall h \in A$ the set function $\lambda \rightarrow h\left[(f \circ G)^{-1}(\lambda)\right]$ is an analytic multivalued function. By this fact and the result obtained by Yamaguchi [7], Nishino [3] et al, we can deduce that the cardinality of $(f \circ G)^{-1}(w)$ is finite for every $w$. The well-known result of Aupetit and Wermer [1] now shows that $G^{-1}(B)$ is a one-dimensional analytic variety; hence $G^{-1}(B) \subset V$ by the maximality of $V$. Thus, $Y \cap G^{-1}(B)=\varnothing$. This is a contradiction, since $z^{*} \in G\left(Y \cap G^{-1}(B)\right)$, which holds because $z^{*} \in f^{-1}\left(w^{*}\right) \cap G(Y) \subset B$. This proves Theorem 1 .

\section{REFERENCES}

1. B. Aupetit and J. Wermer, Capacity and uniform algebras, J. Funct. Anal. 28 (1978), 238400.

2. T. W. Gamelin, On analytic disks in the spectrum of a uniform algebra, Arch. Math. 52 (1989), 269-274.

3. T. Nishino, Sur les ensembles pseudoconcaves, J. Math. Kyoto Univ. (1962), 225-245.

4. Z. Slodkowski, Analytic set-valued functions and spectra, Math. Ann. 256 (1981), 363-386.

5. __ Local maximum property and q-plurisubharmonic functions in uniform algebras, Math. Anal. Appl. 115 (1986), 105-130.

6. J. Wermer, Potential theory and function algebras, Texas Tech. Univ. Math Series, no. 14, 1981, pp. 114-125.

7. H. Yamaguchi, Sur une uniformitte des surfaces contantes d'une fonction entiere de deux varables complexes, J. Math. Kyoto U. 13 (1973), 417-433.

Department of Mathematics, Pennsylvania State University, Berks Campus, Reading, PenNSYlVANia 19610-6009

Department of Mathematics, Statistics and Computer Science, University of Illinois at Chicago, Chicago, Illinois 60680 